\title{
Lifetime reproductive success, selection on lifespan, and multiple sexual ornaments in male European barn swallows
}

\author{
Alessandra Costanzo, ${ }^{1,2}$ Roberto Ambrosini, ${ }^{3}$ Manuela Caprioli, ${ }^{1}$ Emanuele Gatti, ${ }^{4}$ Marco Parolini, ${ }^{1}$ \\ Luca Canova, ${ }^{5}$ Diego Rubolini, ${ }^{1}$ Andrea Romano, ${ }^{1}$ Luca Gianfranceschi, ${ }^{3}$ and Nicola Saino ${ }^{1}$ \\ ${ }^{1}$ Department of Environmental Science and Policy, University of Milan, via Celoria 26, I-20133 Milan, Italy \\ ${ }^{2}$ E-mail: alessandra.costanzo@unimi.it \\ ${ }^{3}$ Department of Earth and Environmental Sciences (DISAT), University of Milano Bicocca, Piazza della Scienza, 1, I-20126 \\ Milan, Italy \\ ${ }^{4}$ Department of Biosciences, University of Milan, via Celoria 26, I-20133 Milan, Italy \\ ${ }^{5}$ Department of Chemistry, Via Taramelli 12, I-27100 Pavia, Italy
}

Received February 7, 2017

Accepted June 30, 2017

\begin{abstract}
Natural and sexual selection arise when individual fitness varies according to focal traits. Extra-pair paternities (EPPs) can affect the intensity of selection by influencing variance in fitness among individuals. Studies of selection require that individual fitness is estimated using proxies of lifetime reproductive success (LRS). However, estimating LRS is difficult in large, open populations where EPPs cause reallocation of biological paternity. Here, we used extensive field sampling to estimate LRS in a population of barn swallows (Hirundo rustica) to estimate selection on lifespan and ornamental traits of males. We found selection on lifespan mediated both by within- and extra-pair fertilization success and selection on tail length mediated by within- but not extra-pair fertilization success. In addition, we found selection on tail white spots via extra-pair fertilization success after controlling for selection on other traits. These results were not confounded by factors that hamper studies of LRS, including nonexhaustive sampling of offspring and biased sampling of males. Hence, natural and sexual selection mediated by LRS operates on lifespan, tail length, and size of the tail white spots in barn swallows.
\end{abstract}

KEY WORDS: Barn swallow, lifetime reproductive success, selection, sperm competition.

Individuals can vary greatly in their relative contribution to the genetic composition of the next generation (Williams 1992). Large variation in individual fitness is most often reflected in the distribution of lifetime reproductive success (LRS) being strongly leftskewed in birds, with most individuals producing few offspring and only a few showing large LRS (Newton 1989; Clutton-Brock 1988). Because variation in individual fitness sets the scope for natural and sexual selection, dissecting the proximate and ultimate sources of variation in LRS is pivotal to our understanding of natural and sexual selection (Williams 1992; Webster et al. 1995; Shuster and Wade 2003).

LRS depends on the combination of the lifetime number of reproductive episodes (e.g., broods) and the average number of viable offspring produced per episode (Newton 1989; McGraw and Caswell 1996; Clutton-Brock 1988). Duration of life can thus be a major source of variation in LRS because it positively affects the lifetime number of reproductive events (Gustafsson 1986; Clutton-Brock 1988; Merilä and Sheldon 2000). However, in some studies, duration of adult lifespan does not strongly predict LRS (Mills 1989; Herényi et al. 2012), suggesting that variations in the number of offspring that are produced in each breeding episode overwhelm lifetime number of breeding episodes in determining LRS.

Variance in male reproductive success can arise in the context of sexual selection processes (Andersson 1994). Sexual selection studies have long sought the proximate and ultimate causes of 
variation in male mating success (Kirkpatrick et al. 1990; Owens and Hartley 1998). Female mate preferences are often nonrandomly distributed with respect to the expression of male secondary sexual traits (Jennions and Petrie 1997; Saino et al. 1997; Møller and Ninni 1998; Griffith et al. 2002; Westneat and Stewart 2003; Wong and Candolin 2005). According to Fisherian/honest indicator mechanisms of sexual selection, such ornamental male traits evolve under the effect of directional intersexual selection by females for traits that reliably signal genetic/phenotypic quality of males and/or predict sexual attractiveness of their future offspring (Weatherhead and Robertson 1979; Andersson 1994; Fawcett et al. 2007). In addition, different male ornamental traits (e.g., skin or feather coloration and courtship displays in fish and birds) typically co-occur and are presented to choosy females simultaneously (Møller and Pomiankowski 1993; Iwasa and Pomiankowski 1994). The selection pressures that lead to the evolution of such "multiple ornaments" and their function in the mate choice process are still contentious issues of debate (Møller and Pomiankowski 1993; Candolin 2003). Importantly, however, conspecific populations may differ in the strength of selection on individual ornaments and this may be a mechanism causing population divergence and eventually speciation (Møller and Cuervo 1998; Panhuis et al. 2001; Van Doorn et al. 2009).

In species where extra-pair paternities (EPPs) occur, variance in reproductive output among males can be affected by the success of males in securing their own (within-pair) paternity of their social progeny (i.e., the offspring generated by their social mate(s)) and in siring extra-pair offspring (EPO) by fertilizing females different from their social mate(s) (Whittingham and Dunn 2005; Lebigre et al. 2012 and references therein). The occurrence of EPPs is most often thought to boost variance in male realized reproductive output because success in siring EPO is not accompanied by a commensurate reduction in the number of within-pair offspring (WPO) (Webster et al. 1995; Sheldon and Ellegren 1999; Vedder et al. 2011). However, depending on the sign of the covariance between within- and extra-pair reproductive success, EPPs may either increase or decrease the variance in reproductive success among males. Hence, competition for paternity via EPP is a potentially double-edged component of sexual selection and can contribute to the evolution of male epigamic traits (Møller and Ninni 1998).

Estimating LRS in iteroparous species is difficult, as it requires long-term studies of populations of individually marked organisms where individuals are monitored during their entire life (Clutton-Brock 1988; Shuster and Wade 2003). The occurrence of frequent EPPs greatly hinders the scope for LRS studies because such studies require collecting exhaustive data on all WPO and EPO sired by individual males. In addition, "edge effects", whereby the study sample reproductively interacts via EPPs with the individuals breeding just outside the study area, can lead to inaccurate LRS estimates due to missed paternity events by the focal males (Webster et al. 1995; Sheldon and Ellegren 1999; Webster et al. 2001). We are unaware of any study of a large, open vertebrate population with frequent EPPs in the wild where the potentially confounding effects of EPP and edge effects could be assumed to have no or negligible role.

The European barn swallow (H. rustica rustica) that we studied is a small migratory passerine bird. These socially monogamous birds breed either as pairs or in colonies of two to tens of pairs, typically in farms, spatially isolated from other colonies in our study area. Females lay 1-3 clutches of 1-7 eggs per breeding season, from April to July (Møller 1994a). The proportion of offspring that are sired by a male different from their social father is high, although temporally and spatially variable (see below), as is the frequency of broods where at least one offspring is sired by an extra-pair male (Møller and Tegelström 1997; Saino et al. 1997; Kojima et al. 2009). Barn swallows are short-lived birds, with most adults having only one breeding season (Møller 1994a). Birds older than 3 years are rare as annual survival of adults is low (0.30-0.40; Møller and de Lope 1999). Importantly, barn swallows of both sexes have extremely high breeding philopatry (Møller 1994a). Hence, birds can be followed throughout their reproductive life, and individuals that do not return to the colony where they bred the previous year can confidently be assumed to have died. In addition, males do not apparently fertilize females breeding in other colonies, at least in our study area (see Ambrosini et al. 2012 for a description of the study area). However, since natal dispersal is high (Balbontín et al. 2009, Scandolara et al. 2014), with the vast majority of yearling recruits immigrating from a colony different from their original one, the frequency of mating between close relatives (parents-offspring; siblings) is extremely low (Kleven et al. 2005), implying that barn swallow populations are not affected by the consequences of inbreeding on population genetic structure.

Here, we identified parentage of all offspring produced at three colonies over three years and measured male LRS (including EPPs) to estimate selection differentials and partial selection differentials (i.e., selection gradients controlling for the effect of selection on correlated traits) on lifespan and a number of male secondary sexual traits that have been shown to have a role in sexual selection and competition for genetic parentage in one or more of the geographical populations/subspecies of this species (Romano et al. 2017). Specifically, using LRS as an estimate of fitness we estimated selection on the length and fluctuating asymmetry of the outermost tail feathers, on the size of the white spots on the tail feathers, and on melanin-based coloration of the white to chestnut ventral plumage region. In addition, we quantified selection on "ordinary" (i.e., nonsexually selected) traits including wing length, which is a major trait affecting flight performance, and body size as gauged by tarsus length. 
Based on several previous studies (review in Romano et al. 2017), we expected that individuals with longer and more symmetric tails, larger white spots on tail feathers, and darker ventral plumage coloration had larger lifetime number of WPO in the broods where they were the social parents $\left(\operatorname{LRS}_{\text {wpo }}\right)$. In addition, we expected that these individuals also sired a larger lifetime number of EPO in broods other than their social broods $\left(\mathrm{LRS}_{\text {epo }}\right)$ and that, as a consequence, they had larger total LRS $\left(\mathrm{LRS}_{\text {tot }}\right.$, corresponding to $\left.\mathrm{LRS}_{\mathrm{wpo}}+\mathrm{LRS}_{\text {epo }}\right)$. In addition, we expected that all indicators of LRS increased with lifespan because the number of breeding events strongly increases with duration of life.

\section{Methods}

We studied barn swallows breeding at three colonies (= farms) located west of Milan (Northern Italy) over five years (20122016). The colonies were chosen to represent very small (3-6 breeding pairs), medium (12-19 breeding pairs), or large-sized (22-26 breeding pairs) according to recent large-scale censuses (Ambrosini et al. 2012). In all study years, we captured and individually marked with numbered metal and plastic colour rings all the adults breeding in the focal colonies. Thanks to the extremely high breeding philopatry, the individuals that were captured in any year between 2013 and 2015 and had not been captured as adults in the previous year could be assumed to be 1-yearold individuals at their first breeding season immigrating from colonies outside our study area, except in rare cases when they were local recruits (i.e., individuals that were ringed as nestlings at the focal colonies allowing us to directly assess age). We could thus a posteriori identify a set of 79 males that started breeding in 2013-2015 and died before 2016. In 2013-2015, all breeding pairs were identified and breeding activities were monitored. Nestlings from all first, second and third broods were ringed and subjected to blood sampling at the age of 8-12 days for parentage analyses.

Standard morphological measurements were taken on all individuals in all capture years, including length of both outermost tail feathers, chord length of both wings, and tarsus length. Tail length and wing length were expressed as the mean of the left and right character. Tail asymmetry was expressed as the unsigned difference between the length of the left and the right outermost tail feathers. Some contour feathers were also collected from the same region of the white to rufous ventral plumage for later spectrometric colour measurements (see below). The fourth (counting outwards) right rectrix (R4) was plucked and stored flat in individual bags for later measurement of the size of the white spot (Saino et al. 2015). A small blood sample was taken for parentage analyses.

\section{SPECTROMETRIC COLOUR ANALYSIS}

Reflectance of one, randomly chosen, ventral feather, was recorded by means of an Avantes DH-2000 spectrometer equipped with a deuterium-tungsten halogen light source in a dark chamber and over a black background, as described in Saino et al. (2013a,b). Coloration was quantified by processing reflectance data according to the tetrahedral colour space model (Goldsmith 1990) using TetraColorSpace program (Version 1a; Stoddard and Prum 2008) implemented in MATLAB 7 (MathWorks, Natick, MA), assuming UVS cone type retina and adopting the spectral sensitivity of the blue tit (Cyanistes caeruleus). Each colour vector in the tetrahedral colour space was then converted into the spherical coordinates $\theta, \varphi$, and rA (Stoddard and Prum 2008). $\theta$ and $\phi$ represent the human visible and the ultraviolet components of chroma, while rA reflects colour saturation. In the range of colours of barn swallow ventral feathers increasing $\theta$ values indicate paler, whitish coloration (Saino et al. 2013a,b). Repeatability of the three coloration variables as estimated by measuring twice the same feather was high ( $r>0.73, n=45$ individuals). Similarly, among-feathers repeatability of the three colour variables estimated by measuring two different feathers from the same region was also high ( $r>0.74, n=10$ individuals) (Saino et al. 2013a). Moreover, as demonstrated in Romano et al. (2015), the reflectance measurement of one ventral feather strongly correlates with the measurements taken on three overlapping feathers and well as directly on the bird's body. The correlation coefficients between the colour variable values obtained by the three measurements were: $\theta: r>0.88, n=14$ individuals; $\phi$ and $\mathrm{rA}: r>0.91$, $n=14$ individuals.

\section{TAIL WHITE SPOT AREA MEASUREMENT}

The R4 feathers were sellotaped to a cardboard backing across the shaft and scanned. Using ImageJ 1.46r software (rsbweb.nih.gov), for each feather we measured the area of the white spot (Saino et al. 2015). Importantly, in an additional sample of birds from which we also plucked the outermost rectrix (R6) (Saino et al. 2015), there was a positive correlation between the area of the white spot on R4 and on the sixth tail feather (R6) ( $r=0.72$, $n=17, P<0.05$ ), indicating that the size of the white spot is correlated within individuals across tail feathers.

\section{GENETIC PARENTAGE ANALYSIS}

DNA was extracted from blood samples by alkaline lysis and diluted to a final concentration of $50 \mathrm{ng} / \mu \mathrm{L}$, according to Saino et al. (2008). Genotyping of adults and nestlings was performed on a total of five loci (see Supporting Information for detailed PCR reaction conditions). Three of them were highly polymorphic microsatellite loci previously developed for barn swallows (Hir7, Hir17, Hir20 (Tsyusko et al. 2007)), one was a microsatellite in the $3^{\prime}$ untranslated region (UTR) of the Adcyap1 gene 
(adenylate cyclase-activating polypeptide 1; original primers Steinmeyer et al. 2009), and one a polymorphic region within the gene for proopiomelanocortin (POMC). POMC primers were designed by the authors using $H$. rustica genomic sequences kindly supplied by Dr. Anne-Lyse Ducrest and Prof. Alexandre Roulin (University of Lausanne, Switzerland; pers. comm. 2015). Either forward or reverse primers were fluorescently labeled (see Table S1). Polymorphism was determined using a commercial fragment analysis service (Macrogen Inc., Seoul, Republic of Korea) (Bazzi et al. 2015). Fragment lengths were scored for each individual using GeneMarker ${ }^{\circledR}$ version 2.4.2 software (Softgenetics). In total, 1046 individuals (235 adults and 811 nestlings) were genotyped at all five loci and 22 (four adults and 18 nestlings) at four loci. Parentage assignment was performed using Cervus version 3.0.3 software. Eight hundred twenty-one of 829 (99.03\%) of the genotyped nestlings could be assigned to their genetic parents (see Supporting Information for details regarding Cervus parentage assignment). The presence of EPP was defined when the genetic father identified by parent pair analyses differed from the social father identified during behavioural observations. In no case did we identify instances of brood parasitism, that is, a nestling that did not genetically match with the social mother.

\section{STATISTICAL ANALYSES}

The phenotypic value of some traits (e.g., plumage traits that are molted annually) in barn swallows can change with age. To obtain an average estimate of the expression of any $i$-th trait for any $j$-th male that bred over more than one year, we first computed the difference $\left(\mathrm{x}_{\mathrm{ijt}}\right)$ between the phenotypic value of the $i$-th trait recorded for the $j$-th individual at age $t$ and the mean population-level phenotypic value of the $i$-th trait recorded on all individuals at age $t$. The phenotypic value pertaining to any $j$-th individual at the $i$-th trait was then computed as the mean $\left(\mathrm{X}_{\mathrm{ij}}\right)$ of all the $\mathrm{x}_{\mathrm{ijt}}$ values recorded for that individual during its entire life. These mean values were then used for the selection analyses detailed below. This procedure to obtain a synthetic phenotypic value while accounting for age effects requires that agecorrected values are significantly repeatable within individuals. In fact, analyses on the raw values of individual traits showed that the intraclass correlation coefficients, reflecting repeatability of traits that are renewed annually, as estimated from variance components of linear mixed models including age as a fixed effect, were large for all traits including tail length $(r=0.74)$, size of white spots on tail ( $r=0.53)$, wing length $(r=0.86)$, and plumage tetrahedral colour components $(\theta: r=0.42 ; \varphi: r=0.67$; rA: $r=0.58$ ) (likelihood-ratio test comparing the model including vs. excluding the random effect of individual identity: all $\chi^{2}$ $\geq 4.63, P \leq 0.03$ ) (see Table 1 for sample sizes). Repeatability of tail asymmetry was estimated in a linear mixed model not including the effect of age, because tail asymmetry did not change with age in the present sample (Kendall's $\tau=-0.03, P=0.72$, $n=98)$ as well as in other samples from the same population (our unpubl. results). Tail asymmetry had repeatability similar in magnitude to that of the other variables, but the statistical test showed that it was marginally nonsignificantly different from 0 ( $r=0.61, \chi^{2}=3.60, P=0.06$ ) (see Table 1 for sample size). Hence, individuals were consistent in their expression of most of the focal phenotypic traits at different ages and their associated phenotypic value could be estimated as the mean of the deviations from the age-specific population means. An exception is represented by tail asymmetry, whose repeatability among years was statistically marginally nonsignificant. The results of the analyses on tail asymmetry should therefore be considered with this caveat in mind. For tarsus length, no adjustment for age was required because this trait does not vary in adulthood, and its phenotypic value at age 1 was therefore used.

To calculate selection gradients, we followed the method by Arnold and Wade (1984). In linear regression analyses of selection on LRS, $\mathrm{X}_{\mathrm{ij}}$ vectors (or tarsus length at age 1) were standardized to a mean $=0$ and variance $=1$. Lifespan was also standardized to mean $=0$ and variance $=1$. Relative fitness, in terms of total number of offspring produced during life by any individual was expressed as the ratio $\left(\mathrm{rLRS}_{\text {tot }}\right)$ between the total offspring fathered divided by the mean number of offspring fathered by the 79 males in the sample. Similarly, the number of WPO or EPO sired was expressed as the ratios $\left(\mathrm{rLRS}_{\mathrm{wpo}}\right.$ or $\mathrm{rLRS}_{\mathrm{epo}}$, respectively) between the WPO or EPO sired by an individual and the mean WPO or EPO sired by the males in the sample. Regression coefficients of rLRS variables on standardized phenotypic traits therefore reflect the proportional change in fitness relative to the population mean caused by a one standard deviation change in the phenotypic trait (Arnold and Wade 1984). The residuals of the regression in some cases did not meet the condition of normality. To test for robustness of the statistical tests, we therefore also applied nonparametric Spearman's correlation analysis. In all cases, the results of regression analyses were qualitatively confirmed by nonparametric correlation analyses, meaning that the tests that were significant with the former approach remained such also with the latter.

Tests for stabilizing/disruptive selection were performed in regression analyses of LRS on second-order polynomial terms on standardized phenotypic traits.

The amount of variance explained by the relationships between rLRS indicators and phenotypic traits was estimated by computing $r^{2}$. Correlation coefficients were compared by $z$-tests.

In the analyses of selection gradients, the significance values of the tests of $\mathrm{rLRS}_{\mathrm{tot}}, \mathrm{rLRS}_{\mathrm{wpo}}$, or $\mathrm{rLRS}_{\mathrm{epo}}$, respectively, were corrected according to the false discovery rate procedure (Benjamini and Hochberg 1995). 


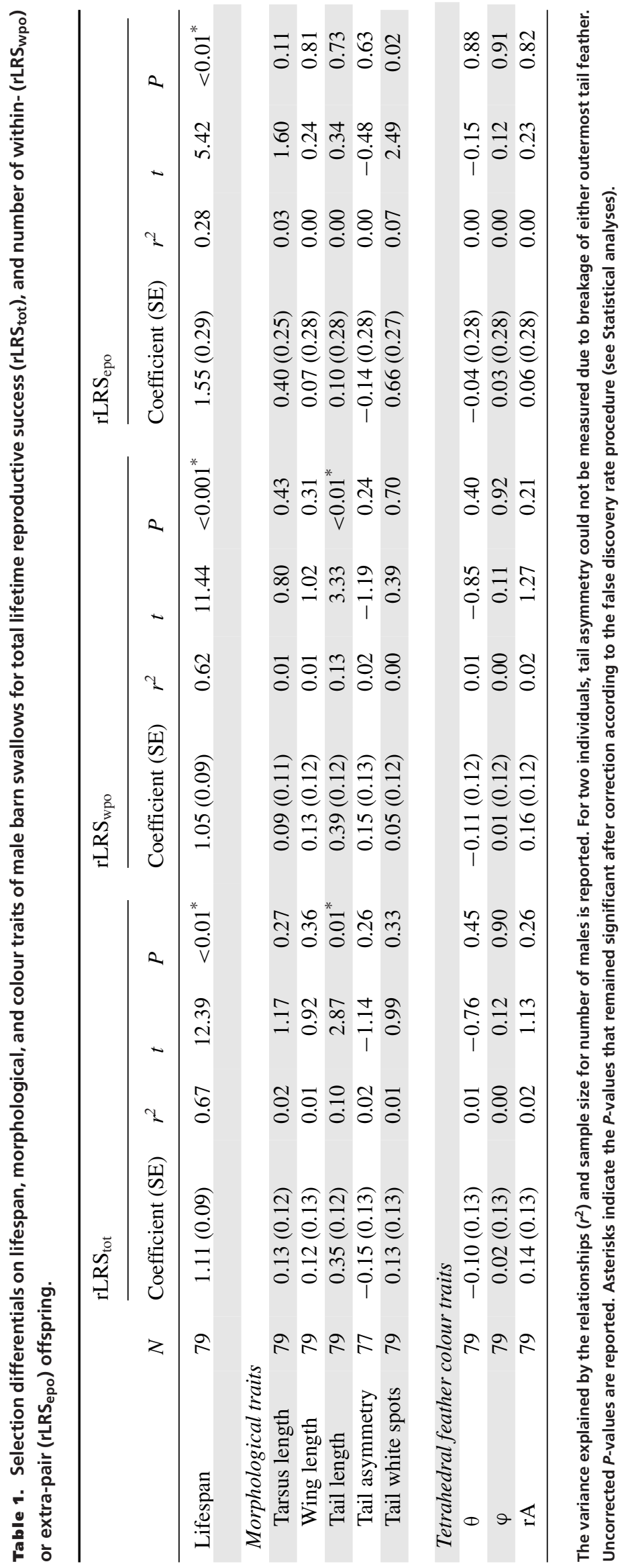


In just one case (lifespan by farm effect on $\mathrm{rLRS}_{\text {epo }}$, see Results), the interaction effect between farm and a phenotypic trait was found to significantly predict rLRS variables after correction of the significance values according to the false discovery rate procedure (Benjamini and Hochberg 1995). In addition, none of the breeding success variables was found to differ among colonies and in all cases the inclusion of the fixed effect of farm in the analyses did not qualitatively alter the results, meaning that the effects that were significant remained such after inclusion of the effect of farm in the model (Table S4). Thus, in all analyses males from the three colonies were pooled.

The analyses were run using PROC GLM in SAS 9.3 or SPSS13. Type III sum of squares was always used. Residuals from regression analyses were visually inspected to assess normality.

Survival in relation to tail length was analyzed in a Cox proportional hazards regression model. In this model, we allowed for time dependency of tail length as a covariate because tail length changes with age. To account for tied event times, we adopted the procedures implemented by PROC PHREG $($ TIES $=$ EXACT $)$ in SAS 9.3, which is based on the probability of the union of the partial likelihoods for all possible orderings of tied events.

Statistical parameters are reported with their associated standard error (SE).

\section{Results}

We measured LRS $\left(\mathrm{LRS}_{\text {tot }}, \mathrm{LRS}_{\mathrm{wpo}}, \mathrm{LRS}_{\text {epo }}\right.$ ) for the 79 males that completed their life-cycle within the study period at the three focal colonies. We assessed parentage of $>99 \%$ of the 829 nestlings that were produced and reached sampling age at the study colonies over the study period. The 79 focal males were found to have sired on average 5.38 (0.66 SE; range: 0-23) WPO nestlings and 0.78 (0.21 SE; range: 0-12) EPO nestlings at the end of their life, yielding a mean total realized reproductive success of $6.16(0.78$ SE; range 0-30) offspring. Thus, of the 487 nestlings that were found to have been sired by the 79 focal males, $425(87.3 \%)$ were WPO while $62(12.7 \%)$ were EPO. The percentage of broods of the 79 focal males where at least one nestling was found to be extra-pair was $34.7 \%$ (58/167 broods). Twenty-one of the 79 males did not produce any WPO nestlings. Ten of these were unmated while 11 males lost their clutch or brood before blood sampling. In three cases, a male that did not produce any WPO produced at least one EPO.

\section{SELECTION DIFFERENTIALS ON LIFETIME REPRODUCTIVE SUCCESS}

Selection on lifespan was large and highly significantly different from 0 for $\mathrm{rLRS}_{\text {tot }}$ (Fig. 1) and also for $\mathrm{rLRS}_{\text {wpo }}$ and $\mathrm{rLRS}_{\mathrm{epo}}$, implying that lifespan is a major determinant of the total number

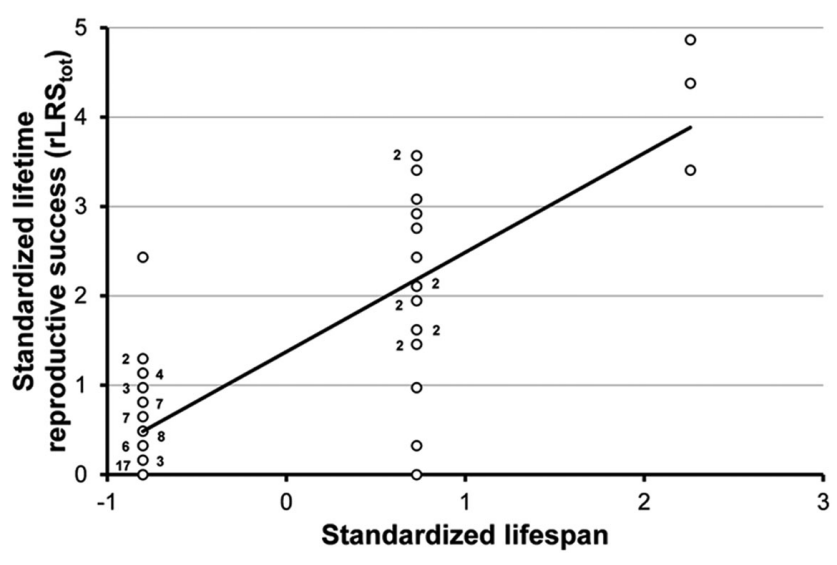

Figure 1. Lifetime reproductive success $\left(\mathrm{rLRS}_{\text {tot }}\right)$ expressed as the ratio between the total number of offspring produced by individual males $(n=79)$ throughout their life and the population mean in relation to lifespan standardized to mean $=0$ and a variance $=$ 1. The slope of the regression line represents the selection differential. The number of overlying data points is indicated. The line is the linear regression line.

of offspring that male barn swallows sire over their life, and that LRS increases with lifespan both as a result of a larger number of WPO and of a larger number of EPO (Table 1). For $\mathrm{rLRS}_{\text {epo }}$, a statistically significant effect of the interaction between lifespan and farm was detected after false discovery rate correction $\left(F_{2,73}=7.14, P<0.01\right)$. The relationship was significantly positive $(P<0.05)$ in the two largest colonies and nonsignificant in the smallest colony. This result suggests that the effect of extra-pair fertilizations on LRS increases with colony size possibly because of more opportunities for EPPs in large colonies.

Selection differentials on nonsexually selected morphological traits (wing length and tarsus length) were not significantly different from 0 for all rLRS components (Table 1). Selection on tail length was positive and strong for the total number of offspring sired (Table 1; Fig. 2) and also for the number of WPO, but not for the number of EPO (Table 1). To test if the slopes of the relationships between $\mathrm{rLRS}_{\mathrm{wpo}}$ or $\mathrm{rLRS}_{\text {epo }}$ and tail length differed we analyzed LRS estimates in a linear model with tail length as a covariate and indicator of LRS ( $\mathrm{rLRS}_{\mathrm{wpo}}$ or $\mathrm{rLRS}_{\mathrm{epo}}$ ) as a classification factor. The interaction effect between indicator of LRS and tail length was not statistically significant $(t=$ 0.97 , df $=154, P=0.33$ ), implying that the slopes of the relationship between LRS and tail length did not differ between $\mathrm{rLRS}_{\text {wpo }}$ and $\mathrm{rLRS}_{\text {epo }}$. However, the correlation coefficients between either indicator of LRS and tail length significantly differed $(z=2.05, P=0.04$ ) (Table 1 ), implying that the relationship with tail length was stronger for $\mathrm{rLRS}_{\mathrm{wpo}}$ than for $\mathrm{LRS}_{\text {epo }}$. However, the increase in either component of fitness per unit increase in tail length did not differ significantly. Importantly, tail 


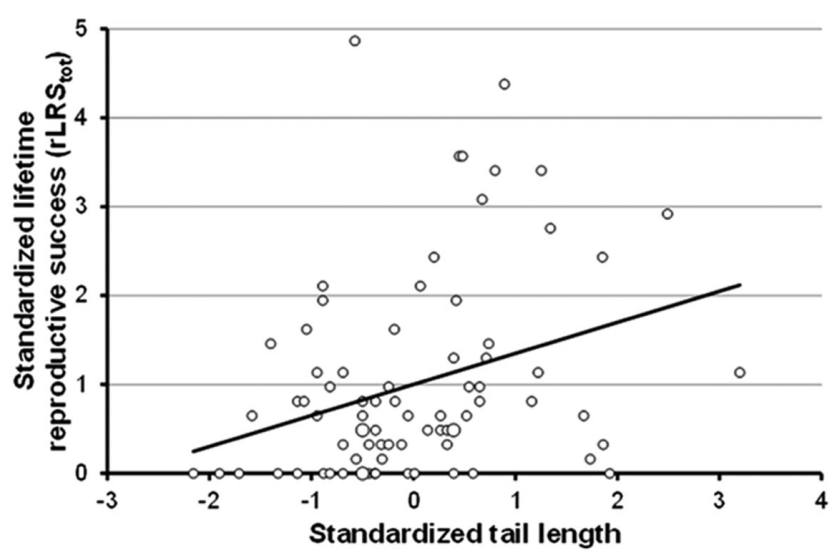

Figure 2. Lifetime reproductive success ( $r L R S_{\text {tot }}$ ) expressed as the ratio between the total number of offspring produced by individual males $(n=79)$ throughout their life and the population mean in relation to tail length corrected for age (see Methods) and standardized to mean $=0$ and a variance $=1$. The slope of the regression line represents the selection differential. Larger dots indicate two overlaying data points. The line is the linear regression line.

length was expressed as the within-individual mean of the residuals from the age-specific mean phenotypic value (see Methods). Tail asymmetry did not significantly predict LRS components (Table 1).

Selection differentials on ventral colour plumage components were weak and not significantly different from 0 for all rLRS components (Table 1). Selection differentials on white tail spots were not significantly different from 0 for $\mathrm{rLRS}_{\text {tot }}$ and $\mathrm{rLRS}_{\mathrm{wpo}}$. Selection differential on white tail spots was marginally nonsignificantly larger than 0 for $\operatorname{rLRS}_{\text {epo }}(P=0.07$ after false discovery rate correction) meaning that there was a marginally nonsignificant trend for individuals with larger white spots on the tail feathers to sire a larger lifetime number of EPO (Table 1; Fig. 3). The slopes of the relationships between $\operatorname{rLRS}_{\mathrm{wpo}}$ or $\mathrm{rLRS}_{\text {epo }}$ and white spots area significantly differed $(t=2.09$, df $=154, P=0.04)$ whereas the correlation coefficients did not ( $z=1.46, P=0.15$ ). Hence, the strength of these relationships did not differ whereas the slopes did so.

Regression analyses of LRS on second-order polynomial terms of phenotypic traits showed no significant effect of the quadratic term after correction of the significance values for false discovery rate. Thus, there was no evidence for stabilizing selection on the traits that we measured.

\section{SELECTION GRADIENTS ON TOTAL LIFETIME REPRODUCTIVE SUCCESS}

We first estimated partial selection differentials (i.e., selection gradients) in multiple regression analyses of $\mathrm{rLRS}_{\text {tot }}$ on lifespan, morphological traits, and $\theta$ colour component. We avoided en-

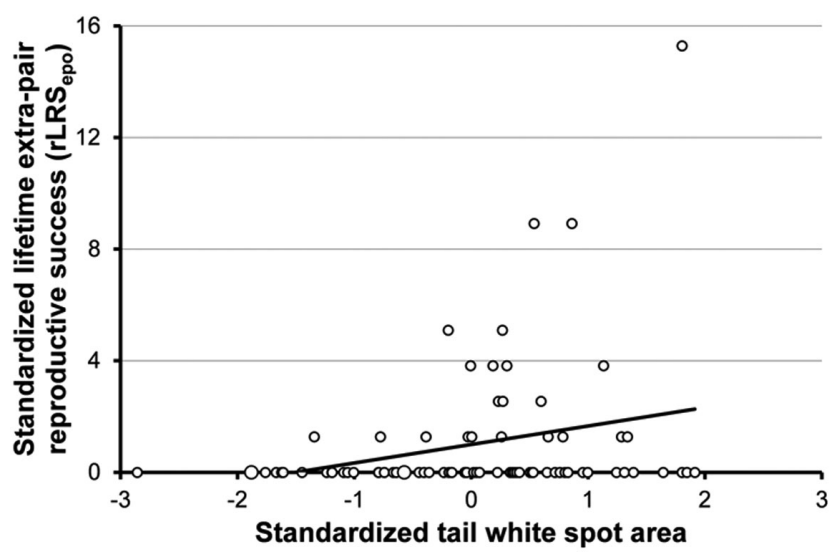

Figure 3. Lifetime extra-pair reproductive success $\left(r L R S_{\text {epo }}\right)$ expressed as the ratio between the total number of extra-pair offspring produced by individual males $(n=79)$ throughout their life and the population mean in relation to size of the white spot on the R4 tail feather corrected for age (see Methods) and standardized to mean $=0$ and a variance $=1$. The slope of the regression line represents the selection differential. Larger dots indicate two overlaying data points. The line is the linear regression line.

tering all three tetrahedral colour components in the same model due to large correlation between the standardized $\theta$ and the rA components $(r=-0.64, n=79, P<0.01)$. The correlations between the other traits were relatively small (unsigned $r$ always smaller than 0.32) suggesting that their simultaneous inclusion in the model did not raise multicollinearity issues. We thus ran separate models where we included only one colour component at a time. The selection gradient was large and significantly larger than 0 for lifespan, showing that longer lived individuals accrue larger fitness independent of their phenotypic traits (Table 2). In addition, the selection gradient was significantly larger than 0 for tail length (Table 2). This implies that long-tailed individuals produce more biological offspring independently of any effect of tail length on survival. Further, tail length was found to positively predict survival in a Cox proportional hazard regression model (coefficient: $-0.05(0.02), \chi^{2}{ }_{1}=6.93, P<0.01$ ). Regression gradients were not significantly different from 0 for tarsus and wing length, tail asymmetry, and the $\theta$ colour component (Table 2). A multiple regression model of $\mathrm{rLRS}_{\mathrm{wpo}}$ confirmed the positive effect of lifespan (Table 2). A multiple regression model of $\mathrm{rLRS}_{\text {epo }}$ disclosed a significant positive effect of tail white spots area (Table 2).

Alternative multiple regression models in which we included the $\varphi$ or rA components of feather coloration were consistent with the model on the $\theta$ component in disclosing significant effects of lifespan and of tail length and in showing nonsignificant effects of the other independent variables on both $\mathrm{rLRS}_{\text {tot }}$ and $\mathrm{rRSS}_{\mathrm{wpo}}$ (other details not shown). In addition, they showed a significant effect of tail white spot area on $\mathrm{LRS}_{\text {epo }}$. 
Table 2. Selection gradients on lifespan, morphological and "visible" plumage chroma for total lifetime reproductive success (rLRS tot $_{\text {). }}$

\begin{tabular}{|c|c|c|c|c|c|c|c|c|c|}
\hline & \multicolumn{3}{|l|}{$\mathrm{rLRS}_{\text {tot }}$} & \multicolumn{3}{|l|}{$\mathrm{rLRS}_{\text {wpo }}$} & \multicolumn{3}{|l|}{$\mathrm{rLRS}_{\text {epo }}$} \\
\hline & Coefficient (SE) & $t$ & $P$ & Coefficient (SE) & $t$ & $P$ & Coefficient (SE) & $t$ & $P$ \\
\hline Lifespan & $1.05(0.09)$ & 11.89 & $<0.01$ & $0.98(0.09)$ & 11.11 & $<0.01$ & $1.50(0.29)$ & 5.13 & $<0.01$ \\
\hline Tarsus length & $-0.05(0.07)$ & -0.46 & 0.51 & $-0.09(0.08)$ & -1.25 & 0.21 & $0.26(0.25)$ & 1.04 & 0.30 \\
\hline Wing length & $0.00(0.08)$ & 0.02 & 0.98 & $0.01(0.08)$ & 0.14 & 0.89 & $-0.06(0.26)$ & -0.23 & 0.82 \\
\hline Tail length & $0.23(0.08)$ & 2.88 & 0.01 & $0.28(0.08)$ & 3.57 & 0.001 & $-0.16(0.26)$ & -0.60 & 0.55 \\
\hline Tail asymmetry & $-0.14(0.08)$ & -1.77 & 0.08 & $-0.12(0.08)$ & -1.61 & 0.11 & $-0.22(0.26)$ & -0.85 & 0.40 \\
\hline Tail white spots & $0.14(0.07)$ & 1.93 & 0.06 & $0.07(0.08)$ & 0.94 & 0.35 & $0.63(0.24)$ & 2.62 & 0.01 \\
\hline$\theta$ & $-0.13(0.07)$ & -1.74 & 0.09 & $-0.13(0.07)$ & -1.82 & 0.07 & $-0.08(0.24)$ & -0.34 & 0.76 \\
\hline
\end{tabular}

The sample included 77 males for which complete phenotypic information was available.

\section{Discussion}

Estimating the intensity of selection on lifespan and sexually selected traits is a key step in the analysis of the evolution of life histories and of sexual selection. Here, based on information on LRS we analyzed selection on lifespan and on secondary sexual and nonsexual morphological traits in male barn swallows while accounting for the effects of EPP. We found evidence for strong LRS directional selection on lifespan and length of the outermost tail feathers while accounting for age-dependent variation in ornamentation. Selection on tail length was independent of the effect of tail length on annual survival. Selection differentials on other sexually selected traits or body size and wing length did not differ significantly from zero. Significant selection based on LRS on tail length was detectable when the within-pair but not the extra-pair component of LRS was considered. The relationship with tail length was stronger for $\mathrm{rLRS}_{\mathrm{wpo}}$ than for $\mathrm{rLRS}_{\mathrm{epo}}$. This implies that selection for larger tail ornaments is significantly different from 0 and is stronger when it is mediated by the number of biological offspring that males can secure in the broods where they are the social fathers rather than via extra-pair fertilizations.

Overall, strong selection existed on lifespan both via the number of WPO and the number of EPO. Interestingly, selection mediated by the number of EPO was significantly larger in the largest colonies. This result was obtained based on a small sample of just three colonies, and should therefore be considered with this caveat in mind. However, it may suggest that selection on lifespan mediated by extra-pair fertilizations is larger in larger colonies, where opportunities for extra-pair fertilizations may be larger and/or more variable among males. Selection on the size of the white spots on the tail was stronger via the lifetime number of EPO compared to WPO. Significant estimates of selection on tail length and size of white spots were not the spurious result of the indirect effect of selection on other traits, including body size and wing length, as implied by the significant selection gradients controlling for these potentially confounding effects.
Selection on lifespan was expected based on the fact that in the barn swallow variance in clutch and brood size is small, fledging success is extremely high and broodedness (i.e., the number of broods per breeding season) varies markedly between yearlings and older individuals (Møller 1994a). Thus, the number of breeding events and, consequently, of offspring produced, markedly increases with the number of breeding seasons that individuals experience (Saino et al. 2012). Notably, there may be exception to the observation of positive selection on lifespan even among short-lived small birds, as suggested by the study of an archipelago population of house sparrows Passer domesticus, where lifespan did not strongly predict realized LRS (Jensen et al. 2004).

While sexual selection has been intensively studied in the barn swallow, including in the present population, for many years (reviews in Romano et al. 2017), this is the first study in which LRS incorporating the effects of extra-pair fertilization has been quantified and estimated for sexual ornaments. Previous studies have shown clearly significant geographical variation in current sexual selection including EPP on individual male ornaments (i.e., melanin-based coloration and tail length) (Møller et al. 2003; Eikenaar et al. 2011; Vortman et al. 2013; Safran et al. 2016; Wilkins et al. 2016; Romano et al. 2017). The present results indicate that in Western Palearctic populations, contrary to what apparently occurs in other Eurasian (Vortman et al. 2011; Hasegawa and Arai 2013) and most Nearctic populations (Safran and McGraw 2004; but see Kleven et al. 2006), tail length is under selection. This observation is consistent with selection due to variance in annual reproductive success (ARS) on tail length, as observed in several Western Palearctic barn swallow populations (e.g., Møller et al. 1998) also while accounting for EPPs (Saino et al. 1997). The area of the white spots on tail feathers was apparently under positive selection mediated by the success in extrapair fertilizations after controlling for selection on other traits. This finding is consistent with the observation that in Northern European population white tail spots are targeted by directional 
sexual selection (Kose et al. 1999), although no previous study has investigated their role in competition for genetic parentage. Conversely, plumage melanin-based coloration, which has been suggested to be under intersexual selection in other subspecies (Romano et al. 2017 and references therein), seems not to be under directional selection mediated by reproductive success in this Italian population. In fact, selection differentials on "human visible" and UV chroma and on colour saturation were far from being statistically significant, and selection gradients controlling for several traits and for lifespan were also statistically nonsignificant. These results are therefore consistent with meta-analytic evidence and individual studies indicating that different plumage ornaments are differently selected in distinct barn swallow subspecies (summarized by Romano et al. 2017). Selection due to variation in LRS on tail length was also accompanied by viability selection, as long-tailed males had larger annual survival, consistent with previous studies (Møller 1994b; Saino et al. 2011).

Selection on tail length may cause evolution in tail length if the prerequisite condition of nonzero additive genetic variation in the trait is met. Previous studies adopting diverse approaches have led to relatively large estimates of heritability in tail length. For example, parent-biological offspring regression analysis has led to narrow sense heritability $\left(h^{2}\right)$ estimates of 0.39 while regression of EPO phenotype on the phenotype of the social (but nonbiological) father have disclosed low and statistically nonsignificant social parent-offspring resemblance ("heritability" estimate: -0.07) (Saino et al. 2003). Such comparison between father and biological versus nonbiological offspring resemblance suggests no significant maternal and environmental effects on father-offspring resemblance and, hence, that large additive genetic variation in tail length exists. In addition, genetic correlations between tail length and other morphological traits including wing length and body size are generally low suggesting that genetic correlations are not expected to constrain the evolutionary change of tail length. Moreover, selection on male tail length has been shown to be consistent in time within populations, though variable in strength among populations (Møller et al. 2006). Significant heritability, weak genetic correlations with other traits, and consistent selection across generations leads to expectations of evolution in male tail length, although no such change seems to have occurred over 25 years (our unpubl. data). This may suggest that environmental effects may mask evolution. For example, deterioration of the ecological conditions in the sub-Saharan wintering quarters of our study population may cause both its current marked demographic decline and an environmental effect consisting in a reduction in the expression of the tail ornaments, which are produced at the time of the single annual molt during winter in Africa (Saino et al. 2004). Indeed, tail length of barn swallows may depend on individual general state at the time of molt (Møller 1994a; Turner 2006), and deterioration of ecological conditions during wintering may hinder the ability of males to grow a long tail. Thus, apparent evolutionary stasis may result from evolutionary increase in tail length being confounded by negative effects of ecological conditions on physiological state and thus on tail growth. Yet an alternative interpretation is that offspring viability negatively covaries with paternal lifespan or LRS. We deem this explanation unlikely, however, because in a recent study we found no relationship between paternal and offspring longevity (Romano et al. 2016; our unpubl. data), and in previous studies we could find no evidence for phenotypic differences at traits that are likely to affect postfledging survival between WPO and EPO (our unpubl. data).

Estimating selection based on variation in LRS in the wild is challenging, because it requires that reproductive performance of individuals is monitored throughout their lives (Lebigre et al. 2012). In addition, one potential pitfall of LRS studies is nonrandom sampling of individuals, because unmated individuals or individuals that fail their breeding attempt early in the breeding cycle may be more likely to go undetected and may not represent a random sample of the population with respect to phenotypic/genetic quality (Sheldon and Ellegren 1999; Webster et al. 2001; Lebigre et al. 2012). In addition, quantifying selection based on variation in LRS is particularly problematic in species where the occurrence of EPPs causes a reallocation of paternity among individuals with respect to the apparent, social mating pattern (Webster et al. 1995). Because variation in success in sperm competition is typically nonrandom with respect to quality of individual males, as reflected for example by their sexual ornaments (Saino et al. 1997; Safran et al. 2005), neglecting the consequences of EPPs on realized LRS can result in biased estimates of selection on sexual ornaments. In the present study, we exhaustively sampled all the offspring and the adults that were present in any study colony during the study period, thereby assessing parentage of more than $99 \%$ of all the offspring produced in the colonies during the study and paying special attention not to exclude individuals that did not breed successfully either because they failed to acquire a social mate or because their breeding attempt failed. Because the genetic father (and mother) could always be identified among the adult males belonging to the focal colonies, extra-pair fertilizations seem not to occur among different colonies, as expected. We can therefore also exclude that our data were confounded by "edge" effects, that is, by missed paternity events by individuals from the focal colonies that fertilized females from other colonies, and be confident that all the biological offspring of the focal males that reached blood sampling age were identified, thereby providing unbiased estimates of realized LRS.

Admittedly, however, our study did not include four- or more years-old individuals because the study spanned five years (20122016), the first year (2012) served to identify the individuals that were 1-year-old recruits in 2013 while the last year (2016) served 
to identify the individuals that had their last breeding season in 2015. However, relatively old (four or more years) individuals are relatively rare (Romano et al. 2016) and in the present sample no males that reached age four years (i.e., were still alive in 2016) were included. While the present results should be considered with this caveat in mind, we are confident that this feature of our data did not markedly bias the results and certainly did not produce spurious evidence of selection for larger lifespan or ornament size because LRS is expected to increase with lifespan and covary positively with tail length.

Notably, the frequency of EPO as estimated by the proportion of offspring sired in broods from other pairs relative to the total number of offspring sired was considerably smaller than in previous studies of the same geographical population (Saino et al. 1997; Møller et al. 1998). We speculate that this could result from a combination of factors. First, barn swallow populations have declined by as much as $50 \%$ during the last decade (Ambrosini et al. 2012), potentially reducing the scope for sperm competition, if sperm competition increases with breeding density (Westneat and Sherman 1997; Møller and Ninni 1998). It should also be noticed that previous EPP estimates were mostly obtained from colonies settled in large cowsheds with a large number of breeding pairs in the same room and typically little physical isolation between nests. This could have boosted the frequency of extra-pair paternity by increasing promiscuity among breeding pairs. Currently, such large colonies have considerably declined in number and no such type of colony is represented in the present sample. It should be emphasized, however, that the colonies where the present study was carried out do not represent exceptions, but, rather, the rule in terms of size and topographical scatter of breeding pairs among farm rooms (Ambrosini et al. 2012), and we deliberately choose the three colonies to represent the whole spectrum of variation between small and relatively large colonies. Second, in a declining population like our focal one the scope for sexual selection may be reduced if decline in population size also entails erosion of additive genetic variance in male quality, thereby reducing the scope for adaptive differential female preference for particular males. Extensive, long-term analysis of variation in the variance in the size of male tail ornaments will provide a clue as to whether reduction in the frequency of EPP is linked to a reduction in the variance in male sexual attractiveness.

In conclusion, we showed that selection currently exists on lifetime and length of the ornamental tail feathers and size of the white spots on the tail but not on other ornaments including ventral plumage coloration or nonsexual traits in a population of the socially monogamous barn swallow. Extra-pair fertilization analysis showed that selection is mediated by certainty of paternity of own social offspring rather than by success in fertilizing extrapair females. These results on selection due to variance in LRS were obtained while controlling for several potentially confound- ing factors, including "edge effects" and nonrandom sampling of the study individuals.

\section{AUTHOR CONTRIBUTIONS}

Conceived the study: AC LG NS. Collected data in the field: RA AC MC MP AR DR NS. Performed feather colour and white spot area measurements: AC NS. Performed parentage analyses: AC MC EG LG MP. Analyzed the data: RA AC NS. Wrote the manuscript: LC AC LG NS.

\section{ACKNOWLEDGMENTS}

We kindly thank A.-L. Ducrest and A. Roulin (University of Lausanne, Switzerland) for supplying barn swallow POMC genomic sequences used for microsatellite primer development. The authors declare no conflicts of interest.

\section{DATA ARCHIVING}

The doi for our data is https://doi.org/10.5061/dryad.7v910.

\section{LITERATURE CITED}

Ambrosini, R., D. Rubolini, P. Trovò, G. Liberini, M. Bandini, A. Romano, C. Sicurella, C. Scandolara, M. Romano, and N. Saino. 2012. Maintenance of livestock farming may buffer population decline of the Barn Swallow Hirundo rustica. Bird Conserv. Int. 22:411-428.

Andersson, M. 1994. Sexual selection. Princeton Univ. Press, Princeton, NJ.

Arnold, S. J., and M. J. Wade. 1984. On the measurement of natural and sexual selection: applications. Evolution 4:720-734.

Balbontín, J., A. P. Møller, I. G. Hermosell, A. Marzal, M. Reviriego, and F. De Lope. 2009. Geographic patterns of natal dispersal in barn swallows Hirundo rustica from Denmark and Spain. Behav. Ecol. Sociobiol. 63:1197-1205.

Bazzi, G., R. Ambrosini, M. Caprioli, A. Costanzo, F. Liechti, E. Gatti, L. Gianfranceschi, S. Podofillini, A. Romano, M. Romano et al. 2015. Clock gene polymorphism and scheduling of migration: a geolocator study of the barn swallow Hirundo rustica. Sci. Rep. 5:12443.

Benjamini, Y., and Y. Hochberg. 1995. Controlling the false discovery rate: a practical and powerful approach to multiple testing. J. R. Stat. Soc. Series B. Stat. Methodol. 57:289-300.

Candolin, U. 2003. The use of multiple cues in mate choice. Biol. Rev. 78:575595.

Clutton-Brock, T. H., ed. 1988. Reproductive success. Univ. of Chicago Press, Chicago, IL.

Eikenaar, C., M. Whitham, J. Komdeur, M. van der Velde, and I. T. Moore. 2011. Testosterone, plumage colouration and extra-pair paternity in male north-american barn swallows. PLoS One 6:e23288

Fawcett, T. W., B. Kuijper, I. Pen and F. J. Weissing. 2007. Should attractive males have more sons? Behav. Ecol. 18:71-80.

Goldsmith, T. H. 1990. Optimization, constraint, and history in the evolution of eyes. Q. Rev. Biol. 65:281-322.

Griffith, S. C., I. P. Owens, and K. A. Thuman. 2002. Extra pair paternity in birds: a review of interspecific variation and adaptive function. Mol. Ecol. 11:2195-2212.

Gustafsson, L. 1986. Lifetime reproductive success and heritability: empirical support for Fisher's fundamental theorem. Am. Nat. 128:761-764.

Hasegawa, M. and E. Arai. 2013. Divergent tail and throat ornamentation in the barn swallow across the Japanese islands. J. Ethol. 31:79-83.

Herényi, M., G. Hegyi, L. Z. Garamszegi, R. Hargitai, G. Michl, B. Rosivall, and J. Török. 2012. Lifetime offspring production in relation to breeding lifespan, attractiveness, and mating status in male collared flycatchers. Oecologia 170:935-942. 
Iwasa, Y., and A. Pomiankowski 1994. The evolution of mate preferences for multiple sexual ornaments. Evolution 48:853-867.

Jennions, M. D., and M. Petrie. 1997. Variation in mate choice and mating preferences: a review of causes and consequences. Biol. Rev. 72:283327.

Jensen, H., B. E. SÆther, T. H. Ringsby, J. Tufto, S. C. Griffith, and H. Ellegren. 2004. Lifetime reproductive success in relation to morphology in the house sparrow Passer domesticus. J. Anim. Ecol. 73:599611

Kirkpatrick, M., T. Price, and S. J. Arnold. 1990. The Darwin-Fisher theory of sexual selection in monogamous birds. Evolution 44:180-193.

Kleven, O., F. Jacobsen, R. J. Robertson, and J. T. Lifjeld. 2005. Extrapair mating between relatives in the barn swallow: a role for kin selection? Biol. Lett. 1:389-392.

Kleven, O., F. Jacobsen, R. Izadnegahdar, R. J. Robertson, and J. T. Lifjeld, J. T. 2006. Male tail streamer length predicts fertilization success in the North American barn swallow (Hirundo rustica erythrogaster). Behav. Ecol. Sociobiol. 59:412-418.

Kojima W., W. Kitamura, S. Kitajima, Y. Ito, K. Ueda, G. Fujita, and H. Higuchi. 2009. Female barn swallows gain indirect but not direct benefits through social mate choice. Ethology 115:939-947.

Kose, M., R. Mänd, and A. P. Møller. 1999. Sexual selection for white tail spots in the barn swallow in relation to habitat choice by feather lice. Anim. Behav. 58:1201-1205.

Lebigre, C., P. Arcese, R. J. Sardell, L. F. Keller, and J. M. Reid. 2012. Extra-pair paternity and the variance in male fitness in song sparrows (Melospiza melodia). Evolution 66:3111-3129.

McGraw, J. B. and H. Caswell. 1996. Estimation of individual fitness from life-history data. Am. Nat. 147:47-64.

Merilä, J., and B. C. Sheldon. 2000. Lifetime reproductive success and heritability in nature. Am. Nat. 155:301-310.

Mills, J. A. 1989. Red-billed gull. Pp. 387-404 in I. Newton, ed. Lifetime reproduction in birds. Academic Press, Lond. and San Diego, CA.

Møller, A. P. 1994a. Sexual selection and the barn swallow. Oxford Univ. Press, Oxford, U.K.

1994b. Male ornament size as a reliable cue to enhanced offspring viability in the barn swallow. Proc. Natl. Acad. Sci. U. S. A. 91:69296932.

Møller, A. P., and J. J. Cuervo. 1998. Speciation and feather ornamentation in birds. Evolution 52:859-869.

Møller, A. P., and F. De Lope. 1999. Senescence in a short-lived migratory bird: age-dependent morphology, migration, reproduction and parasitism. J. Anim. Ecol. 68:163-171.

Møller A. P., and P. Ninni. 1998. Sperm competition and sexual selection: a meta-analysis of paternity studies of birds. Behav. Ecol. Sociobiol. 43:345-358.

Møller, A. P. and A. Pomiankowski. 1993. Why have birds got multiple sexual ornaments? Behav. Ecol. Sociobiol. 32:167-176.

Møller, A. P., and H. Tegelström. 1997. Extra-pair paternity and tail ornamentation in the barn swallow Hirundo rustica. Behav. Ecol. Sociobiol. 41:353-360.

Møller, A. P., A. Barbosa, J. J. Cuervo, F. de Lope, S. Merino, and N. Saino. 1998. Sexual selection and tail streamers in the barn swallow. Proc. R. Soc. Lond. Ser. B Biol. Sci. 265:409-414.

Møller, A. P., J. Brohede, J. J. Cuervo, F. de Lope and C. R. Primmer. 2003. Extrapair paternity in relation to sexual ornamentation, arrival date and condition in a migratory bird. Behav. Ecol. 14:707-712.

Møller, A. P., Y. Chabi, J. J. Cuervo, F. de Lope, J. Kilpimaa, M. Kose, P. Matyjasiak, P. L. Pap, N. Saino, R. Sakraoui et al. 2006. An analysis of continent-wide patterns of sexual selection in a passerine bird. Evolution 60:856-868.
Newton, I., ed. 1989. Lifetime reproduction in birds. Academic Press, Lond.

Owens, I. P. F., and I. R. Hartley. 1998. Sexual dimorphism in birds: why are there so many different forms of dimorphism? Proc. R. Soc. Lond. Ser. B Biol. Sci. 265:397-407.

Panhuis, T. M., R. Butlin, M. Zuk, and T. Tregenza. 2001. Sexual selection and speciation. Trends Ecol. Evol. 16:364-371.

Romano, A., M. Romano, M. Caprioli, A. Costanzo, M. Parolini, D. Rubolini, and N. Saino. 2015. Sex allocation according to multiple sexually dimorphic traits of both parents in the barn swallow (Hirundo rustica). J. Evol. Biol. 28:1234-1247.

Romano, A., A. Costanzo, D. Rubolini, N. Saino, and A. P. Møller. 2017. Geographical and seasonal variation in the intensity of sexual selection in the barn swallow Hirundo rustica: a meta-analysis. Biol. Rev. 9: $1582-1600$.

Romano, A., A. Costanzo, M. Caprioli, M. Parolini, R. Ambrosini, D. Rubolini, and N. Saino. 2016. Better-surviving barn swallow mothers produce more and better-surviving sons. Evolution 70:1120-1128.

Safran, R. J., and K. J. McGraw. 2004. Plumage coloration, not length or symmetry of tail-streamers, is a sexually selected trait in North American barn swallows. Behav. Ecol. 15:455-461.

Safran, R. J., C. R. Neuman, K. J. McGraw, and I. J. Lovette. 2005. Dynamic paternity allocation as a function of male plumage color in barn swallows. Science 309:2210-2212.

Safran, R. J., Y. Vortman, B. R. Jenkins, J. K. Hubbard, M. R. Wilkins, R. J. Bradley, and A. Lotem. 2016. The maintenance of phenotypic divergence through sexual selection: an experimental study in barn swallows Hirundo rustica. Evolution 70:2074-2084.

Saino, N., C. R. Primmer, H. Ellegren, and A. P. Møller. 1997. An experimental study of paternity and tail ornamentation in the barn swallow (Hirundo rustica). Evolution 51:562-570.

Saino, N., R. Martinelli, M. Romano, and A. P. Møller. 2003. High heritable variation of a male secondary sexual character revealed by extra-pair fertilization in the barn swallow. Ital. J. Zool. 70:167-174.

Saino, N., T. Szép, R. Ambrosini, M. Romano, and A. P. Møller. 2004. Ecological conditions during winter affect sexual selection and breeding in a migratory bird. Proc. R. Soc. Lond. Ser. B Biol. Sci. 271:681-686.

Saino, N., R. M. De Ayala, G. Boncoraglio, and R. Martinelli. 2008. Sex difference in mouth coloration and begging calls of barn swallow nestlings. Anim. Behav. 75:1375-1382.

Saino, N., M. Caprioli, M. Romano, G. Boncoraglio, D. Rubolini, R. Ambrosini, A. Bonisoli-Alquati, and A. Romano. 2011. Antioxidant defenses predict long-term survival in a passerine bird. PLoS One 6:e19593.

Saino, N., M. Romano, R. Ambrosini, D. Rubolini, G. Boncoraglio, M. Caprioli, and A. Romano. 2012. Longevity and lifetime reproductive success of barn swallow offspring are predicted by their hatching date and phenotypic quality. J. Anim. Ecol. 81:1004-1012.

Saino, N., M. Romano, D. Rubolini, C. Teplitsky, R. Ambrosini, M. Caprioli, L. Canova, and K. Wakamatzu. 2013a. Sexual dimorphism in melanin pigmentation, feather coloration and its heritability in the barn swallow (Hirundo rustica). PLoS One 8:e58024.

Saino, N., M. Romano, D. Rubolini, R. Ambrosini, M. Caprioli, A. Milzani, A. Costanzo, G. Colombo, L. Canova, and K. Wakamatzu. 2013b. Viability is associated with melanin-based coloration in the barn swallow (Hirundo rustica). PLoS One 8:e60426.

Saino, N., M. Romano, A. Romano, D. Rubolini, R. Ambrosini, M. Caprioli, M. Parolini, C. Scandolara, G. Bazzi, and A. Costanzo. 2015. White tail spots in breeding Barn Swallows Hirundo rustica signal body condition during winter moult. Ibis 157:722-730.

Scandolara, C., R. Lardelli, G. Sgarbi, M. Caprioli, R. Ambrosini, D. Rubolini, and N. Saino. 2014. Context-, phenotype-, and kin-dependent natal 
dispersal of barn swallows (Hirundo rustica). Behav. Ecol. 25:180190.

Sheldon, B. C., H. Ellegren. 1999. Sexual selection resulting from extrapair paternity in collared flycatchers. Anim. Behav. 57:285-298.

Shuster, S. M., and M. J. Wade. 2003. Mating systems and strategies. Princeton Univ. Press, Princeton, NJ.

Steinmeyer, C., J. C. Mueller, and B. Kempenaers. 2009. Search for informative polymorphisms in candidate genes: clock genes and circadian behaviour in blue tits. Genetica. 136:109-117.

Stoddard, M. S., and R. O. Prum. 2008. Evolution of avian plumage color in a tetrahedral color space: a phylogenetic analysis of new world buntings. Am. Nat. 171:755-776.

Tsyusko, O. V., M. B. Peters, C. Hagen, T. D. Tuberville, T. A. Mousseau, A. P. Møller, and T. C. Glenn. 2007. Microsatellite markers isolated from barn swallows (Hirundo rustica). Mol. Ecol. Notes 7:833-835.

Turner, A. 2006. The Barn Swallow. T \& A D Poyser, London.

Van Doorn, G. S., P. Edelaar, and F. J. Weissing. 2009. On the origin of species by natural and sexual selection. Science 326:1704-1707.

Vedder, O., J. Komdeur, M. van der Velde, E. Schut, and M. J. Magrath. 2011. Polygyny and extra-pair paternity enhance the opportunity for sexual selection in blue tits. Behav. Ecol. Sociobiol. 65:741-752.

Vortman, Y., A. Lotem, R. Dor, I. J. Lovette, and R. J. Safran. 2011. The sexual signals of the East-Mediterranean barn swallow: a different swallow tale. Behav. Ecol. 22:1344-1352.

. 2013. Multiple sexual signals and behavioral reproductive isolation in a diverging population. Am. Nat. 182:514-523.
Weatherhead, P. J. and R. J. Robertson. 1979. Offspring quality and the polygyny threshold: 'the sexy son hypothesis'. Am. Nat. 113:201-208.

Webster, M. S., S. Pruett-Jones, D. F. Westneat, and S. J. Arnold. 1995. Measuring the effects of pairing success, extra-pair copulations and mate quality on the opportunity for sexual selection. Evolution 49:1147-1157.

Webster, M. S., H. C. Chuang-Dobbs, and R. T. Holmes. 2001. Microsatellite identification of extra-pair sires in a socially monogamous warbler. Behav. Ecol. 12:439-446.

Westneat, D. F., and P. W. Sherman. 1997. Density and extra-pair fertilizations in birds: a comparative analysis. Behav. Ecol. Sociobiol. 41:205-215.

Westneat, D. F., and I. R. Stewart. 2003. Extra-pair paternity in birds: causes, correlates, and conflict. Annu. Rev. Ecol. Evol. Syst. 34:365-396.

Whittingham, L. A., and P. O. Dunn. 2005. Effects of extra-pair and within-pair reproductive success on the opportunity for selection in birds. Behav. Ecol. 16:138-144.

Wilkins, M. R., H. Karaardıç, Y. Vortman, T. L. Parchman, T. Albrecht, A. Petrželková, L. Özkan, P. L. Pap, J. K. Hubbard, A. K. Hund et al. 2016. Phenotypic differentiation is associated with divergent sexual selection among closely related barn swallow populations. J. Evol. Biol. 29:24102421.

Williams, G. C. 1992. Natural selection. Oxford Univ. Press, Oxford, U.K.

Wong, B., and U. Candolin, U. 2005. How is female mate choice affected by male competition? Biol. Rev. 80:559-571.

Associate Editor: S. Baird Handling Editor: P. Tiffin

\section{Supporting Information}

Additional Supporting Information may be found in the online version of this article at the publisher's website:

Table S1. Sequences and labelling of primers used to genotype adults and nestlings.

Table S2. Statistics for microsatellite loci used to determine paternity in barn swallows.

Table S3. Combined non-exclusion probability for first and second parent calculated for each year and each colony.

Table S4. Selection differentials on lifespan, morphological and colour traits of male barn swallows for total lifetime reproductive success (rLRS tot ), and number of within- $\left(\mathrm{rLRS}_{\mathrm{wpo}}\right)$ or extra-pair $\left(\mathrm{rLRS}_{\mathrm{epo}}\right)$ offspring. 